Bull. Korean Math. Soc. 51 (2014), No. 6, pp. 1841-1850

http://dx.doi.org/10.4134/BKMS.2014.51.6.1841

\title{
ON THE $m$-POTENT RANKS OF CERTAIN SEMIGROUPS OF ORIENTATION PRESERVING TRANSFORMATIONS
}

\author{
Ping Zhao, Taijie You, and Huabi Hu
}

\begin{abstract}
It is known that the ranks of the semigroups $\mathcal{S O P}{ }_{n}, \mathcal{S P O P}{ }_{n}$ and $\mathcal{S} \mathcal{S P O P}{ }_{n}$ (the semigroups of orientation preserving singular selfmaps, partial and strictly partial transformations on $X_{n}=\{1,2, \ldots, n\}$, respectively) are $n, 2 n$ and $n+1$, respectively. The idempotent rank, defined as the smallest number of idempotent generating set, of $\mathcal{S O P}_{n}$ and $\mathcal{S} \mathcal{S P O P}{ }_{n}$ are the same value as the rank, respectively. Idempotent can be seen as a special case (with $m=1$ ) of $m$-potent. In this paper we investigate the $m$-potent ranks, defined as the smallest number of $m$ potent generating set, of the semigroups $\mathcal{S O P}_{n}, \mathcal{S P O P}{ }_{n}$ and $\mathcal{S} \mathcal{S P O P}{ }_{n}$. Firstly, we characterize the structure of the minimal generating sets of $\mathcal{S O P}_{n}$. As applications, we obtain that the number of distinct minimal generating sets is $(n-1)^{n} n$ !. Secondly, we show that, for $1 \leq m \leq n-1$, the $m$-potent ranks of the semigroups $\mathcal{S O P}{ }_{n}$ and $\mathcal{S P O P}{ }_{n}$ are also $n$ and $2 n$, respectively. Finally, we find that the 2-potent rank of $\mathcal{S} \mathcal{S P O P}{ }_{n}$ is $n+1$.
\end{abstract}

\section{Introduction and preliminaries}

As usual we denote by $\mathcal{P} \mathcal{T}_{n}$ the monoid of all partial transformations of a finite set $X_{n}$ with $n$ elements (under composition), by $\mathcal{T}_{n}$ the submonoid of $\mathcal{P} \mathcal{T}_{n}$ of all full transformations of $X_{n}$ and by $\mathcal{S}_{n}$ the symmetric group on $X_{n}$, i.e., the subgroup of $\mathcal{P} \mathcal{T}_{n}$ of all injective full transformations (permutations) of $X_{n}$. Denote by $\mathcal{S P} \mathcal{T}_{n}$ the semigroup of $\mathcal{P} \mathcal{T}_{n} \backslash \mathcal{S}_{n}$ of all singular partial transformations and by $\mathcal{S i n g}_{n}$ the semigroup $\mathcal{T}_{n} \backslash \mathcal{S}_{n}$ of all singular transformations of $X_{n}$.

Let now $X_{n}$ be a chain with $n$ elements, say $X_{n}=\{1<2<\cdots<n\}$. Let $c=\left(c_{1}, c_{2}, \ldots, c_{t}\right)$ be a sequence of $t(t \geq 0)$ elements from the chain $X_{n}$. We say that $c$ is cyclic if there exists no more than one index $i \in\{1, \ldots, t\}$ such that $c_{i}>c_{i+1}$, where $c_{t+1}$ denotes $c_{1}$. Let $\alpha \in \mathcal{P} \mathcal{T}_{n}$ and suppose that

Received November 28, 2013; Revised February 23, 2014.

2010 Mathematics Subject Classification. 20M20, $20 \mathrm{M} 10$.

Key words and phrases. transformation, orientation-preserving, rank, idempotent rank, $m$-potent rank.

This work is supported by Natural Science Foundation of Guizhou (No.[2013]2225) and National Natural Science Foundation of China (No. 11461014). 
$\operatorname{dom}(\alpha)=\left\{a_{1}, \ldots, a_{t}\right\}$, with $t \geq 0$ and $a_{1}<\cdots<a_{t}$. We say that $\alpha$ is orientation-preserving if the sequence of its image $\left(a_{1} \alpha, \ldots, a_{t} \alpha\right)$ is cyclic. Denote by $\mathcal{S P O P} \mathcal{P}_{n}$ the subsemigroup of $\mathcal{S P} \mathcal{T}_{n}$ of all partial orientation preserving singular transformations, by $\mathcal{S O P}{ }_{n}$ the subsemigroup $\mathcal{S P O P}{ }_{n} \cap \mathcal{S i n g}_{n}$ of all orientation preserving singular transformations and by $\mathcal{S} \mathcal{S P O P}{ }_{n}$ the subsemigroup $\mathcal{S P O P} \mathcal{P}_{n} \backslash \mathcal{S O P}{ }_{n}$ of all orientation preserving strictly partial transformations.

Remark 1. In this paper it will always be clear from context when additions are modular.

An element $a$ of any given semigroup is called $m$-potent if $a^{m+1}=a^{m}$ and $a, a^{2}, \ldots, a^{m}$ are distinct. In particular, we refer to idempotent as 1-potent. This concept was mentioned in $[1,6,7]$.

As usual, the rank of a finite semigroup $S$ is defined by $\operatorname{rank} S=\min \{|A| \mid$ $A \subseteq S,\langle A\rangle=S\}$. If $S$ is generated by its set $E$ of idempotents, then the idempotent rank of $S$ is defined by idrank $S=\min \{|A| \mid A \subseteq E,\langle A\rangle=S\}$. If $S$ is generated by its set $E_{m}$ of $m$-potents, then the $m$-potent rank of $S$ is defined by $\operatorname{rank}_{m} S=\min \left\{|A| \mid A \subseteq E_{m},\langle A\rangle=S\right\}$.

It is known that the ranks of the semigroups $\mathcal{S O P}{ }_{n}, \mathcal{S P O P}{ }_{n}$ and $\mathcal{S S P O P}{ }_{n}$ are $n, 2 n$ and $n+1$, respectively. The idempotent rank of $\mathcal{S O P}{ }_{n}$ and $\mathcal{S P O P}{ }_{n}$ are the same value as the rank, respectively (see [8]). In this paper, we investigate the $m$-potent ranks of the semigroup $\mathcal{S O P}_{n}, \mathcal{S P O P}{ }_{n}$ and $\mathcal{S S P O P}_{n}$. In Section 2 we characterize the structure of the minimal generating sets of $\mathcal{S O P}_{n}$. As applications, we prove that the number of distinct minimal generating sets is $(n-1)^{n} n !$. Moreover, we show that, for $1 \leq m \leq n-1$, the $m$-potent ranks of the semigroups $\mathcal{S O P}{ }_{n}$ and $\mathcal{S P O P}{ }_{n}$ are $n$ and $2 n$, respectively. In Section 3 we find that the 2-potent rank of $\mathcal{S S P O P}{ }_{n}$ is $n+1$.

Let $\alpha \in \mathcal{P} \mathcal{T}_{n}$. As usual, we write $\operatorname{im}(\alpha)$ and $\operatorname{rank}(\alpha)$ for the image of $\alpha$ and the rank of $\alpha$, respectively. (The rank of a transformation is defined to be the size of its image.) The kernel of $\alpha$ is the equivalence $\operatorname{ker}(\alpha)=\{(x, y) \in$ $\left.X_{n} \times X_{n}: x \alpha=y \alpha\right\}$. From Fernandes, Gomes, and Jesus [3], Green's relations on $\mathcal{S P O P}{ }_{n}$ are characterized by

$$
\begin{aligned}
& \alpha \mathcal{L} \beta \text { if and only if } \operatorname{im}(\alpha)=\operatorname{im}(\beta), \\
& \alpha \mathcal{R} \beta \text { if and only if } \operatorname{ker}(\alpha)=\operatorname{ker}(\beta), \\
& \alpha \mathcal{J} \beta \text { if and only if } \operatorname{rank}(\alpha)=\operatorname{rank}(\beta) .
\end{aligned}
$$

The semigroup $\mathcal{S P O P}{ }_{n}$ has $n$ J-classes, namely $J_{0}, J_{1}, \ldots, J_{n-1}$, where $J_{0}$ consists of the empty mapping and $J_{r}=\left\{\alpha \in \mathcal{S P O P P} \mathcal{P}_{n}: \operatorname{rank}(\alpha)=r\right\}$ for $1 \leq r \leq n-1$. For $0 \leq r \leq s \leq n$, let

$$
[s, r]=\left\{\alpha \in \mathcal{S P O P} \mathcal{P}_{n}:|\operatorname{dom}(\alpha)|=s, \operatorname{rank}(\alpha)=r\right\} .
$$

Then $J_{r}=\cup_{i=r}^{n}[i, r]$ and $J_{n-1}=[n, n-1] \cup[n-1, n-1]$. We draw attention to the top $\mathcal{J}_{\text {-class }} J_{n-1}$. As in [8] we use the notation

$$
L_{k}=\left\{\alpha \in J_{n-1}: \operatorname{im}(\alpha)=X_{n} \backslash\{k\}\right\},
$$


$R_{(k, k+1)}=\{\alpha \in[n, n-1]:$ the unique non-singleton class of $\operatorname{ker}(\alpha)$ is $\{k, k+1\}\}$ and

$$
R_{k}=\left\{\alpha \in[n-1, n-1]: \operatorname{dom}(\alpha)=X_{n} \backslash\{k\}\right\}, k \in X_{n}
$$

for $\mathcal{L}$-classes and $\mathcal{R}$-classes in $J_{n-1}$. Hence $J_{n-1}$ has $n \mathcal{L}$-classes $L_{1}, L_{2}, \ldots, L_{n}$ and $2 n \mathcal{R}$-classes $R_{(1,2)}, R_{(2,3)}, \ldots, R_{(n, 1)}, R_{1}, R_{2}, \ldots, R_{n}$.

Gomes and Howie [4] used the notation $[i \rightarrow i-1]$ for the idempotent $e$ defined by $i e=i-1, x e=x(x \neq i)$ and the notation $[i \rightarrow i+1]$ for the idempotent $f$ defined by if $=i+1, x f=x(x \neq i)$. They also used the notation $\delta_{k}, k=1,2, \ldots, n$, for the identity mapping on $X_{n} \backslash\{k\}$.

Let $S$ be a subset of $\mathcal{S P O P}{ }_{n}$. As usual, we denote by $E(S)$ the set of all idempotents of $S$. Employing the above notation, the set $E\left(J_{n-1}\right)$ consists of $n$ decreasing idempotents $[i \rightarrow i-1]\left(i \in X_{n}\right), n$ increasing idempotents $[i \rightarrow i+1]\left(i \in X_{n}\right)$ and $n$ idempotents $\delta_{k}$. Notice that $[1 \rightarrow 0]=[1 \rightarrow n]$, $[n \rightarrow n+1]=[n \rightarrow 1]$, etc., by Remark 1. Let $E_{n-1}^{+}=\left\{[i \rightarrow i+1]: i \in X_{n}\right\}$ and $E_{n-1}^{-}\left\{[i+1 \rightarrow i]: i \in X_{n}\right\}$ be the increasing and decreasing idempotent set of $[n, n-1]$, respectively, and let $F_{n-1}=\left\{\delta_{1}, \ldots, \delta_{n}\right\}$. Then $E\left(J_{n-1}\right)=$ $E_{n-1}^{+} \cup E_{n-1}^{-} \cup F_{n-1}$.

Given a subset $U$ of a semigroup $S$ and $s \in S$, we denote by $L_{s}, R_{s}$ and $H_{s}$ the $\mathcal{L}$-class, $\mathcal{R}$-class and $\mathcal{H}$-class of $s$, respectively. For general background on Semigroup Theory, we refer the reader to Howie's book [5].

\section{The $m$-potent ranks of $\mathcal{S O P}_{n}$ and $\mathcal{S P O P}_{n}$}

In this section we characterize the structure of the minimal generating sets of $\mathcal{S O P}{ }_{n}$. As applications, we prove that the number of distinct minimal generating sets is $(n-1)^{n} n$ !. Moreover, we show that, for $1 \leq m \leq n-1$, the $m$ potent ranks of the semigroups $\mathcal{S O P}_{n}$ and $\mathcal{S P O P}$ are $n$ and $2 n$, respectively.

We begin by recalling that Zhao, Xu and Yang [9, Theorem 2.1] proved:

Lemma 2.1. Let $n \geq 3$. Let $G \subseteq E\left(\mathcal{S O P}_{n}\right)$. Then

$$
\langle G\rangle=\mathcal{S O} \mathcal{P}_{n} \text { if and only if } E_{n-1}^{+} \subseteq G \text { or } E_{n-1}^{-} \subseteq G .
$$

For any $i, j \in X_{n}$, let

$$
H_{(i, i+1)}^{[j]}=R_{(i, i+1)} \cap L_{j} \text { and } H_{(i)}^{[j]}=R_{i} \cap L_{j} .
$$

Notice that each $\mathcal{H}$-classes contained in $[n, n-1]$ has the form $H_{(i, i+1)}^{[j]}$, for some $i, j \in X_{n}$, and each $\mathcal{H}$-classes contained in $[n-1, n-1]$ has the form $H_{(i)}^{[j]}$, for some $i, j \in X_{n}$. With above notation, we have the following simple observation:

Lemma 2.2. Let $n \geq 3$. Then

$$
\begin{gathered}
H_{(i, i+1)}^{[k]} H_{(j, j+1)}^{[l]}=H_{(i, i+1)}^{[l]}, \text { if } k=j \text { or } k=j+1, \\
H_{(i)}^{[k]} H_{(j, j+1)}^{[l]}=H_{(i)}^{[l]}, \text { if } k=j \text { or } k=j+1,
\end{gathered}
$$




$$
H_{(k)}^{[i]} H_{(l)}^{[j]}=H_{(k)}^{[j]}, \text { if } i=l .
$$

Notice that $\mathcal{S O P}{ }_{n} \cap J_{n-1}=[n, n-1]$ and $[n, n-1]$ contains $n \mathcal{R}$-classes and $n \mathcal{L}$-classes of $\mathcal{S O P}_{n}$. Thus the number of $\mathcal{R}$-classes and $\mathcal{L}$-classes of $\mathcal{S O P}_{n}$ contained in $[n, n-1]$ are both equal to $n$.

Lemma 2.3. Let $n \geq 3$, and let $A$ be a nonempty subset of $\mathcal{S O P}_{n}$ with $n$ elements. If $A$ contains exactly one element from each $\mathcal{R}$-class and from each $\mathcal{L}$-class of $\mathcal{S O P}_{n}$ contained in $[n, n-1]$, then $\mathcal{S O P}_{n}=\langle A\rangle$.

Proof. By Lemma 2.1, we have $\mathcal{S O P}{ }_{n}=\left\langle E_{n-1}^{+}\right\rangle$. Notice that $A \subseteq[n, n-1] \subseteq$ $\mathcal{S O P}_{n}$. We shall show that $E_{n-1}^{+} \subseteq\langle A\rangle$ and so $\mathcal{S O P}_{n}=\langle A\rangle$. Let

$E_{n-1}^{+}(A)=\left\{[i \rightarrow i+1] \in E_{n-1}^{+}:\right.$there exists $\alpha \in A$ such that $\left.\alpha \mathcal{H}[i \rightarrow i+1]\right\}$ and

$$
X_{n}(A)=\left\{i \in X_{n}:[i \rightarrow i+1] \notin E_{n-1}^{+}(A)\right\} .
$$

Let $[i \rightarrow i+1] \in E_{n-1}^{+}$. If $[i \rightarrow i+1] \in E_{n-1}^{+}(A)$, then there exists $\alpha \in A$ such that $\alpha \mathcal{H}[i \rightarrow i+1]$. Since $[i \rightarrow i+1]$ is an idempotent, it follows that $[i \rightarrow i+1]=$ $\alpha^{\omega}$ for some $\omega \in \mathbb{N}$, and so $[i \rightarrow i+1] \in\langle A\rangle$. If $[i \rightarrow i+1] \in E_{n-1}^{+} \backslash E_{n-1}^{+}(A)$, then $i \in X_{n}(A)$ and, by the property of $A$, there exists $i_{1} \in X_{n}(A) \backslash\{i\}$ such that

$$
\alpha_{i_{1}} \in A \text { and }[i \rightarrow i+1] \mathcal{L} \alpha_{i_{1}} \mathcal{R}\left[i_{1} \rightarrow i_{1}+1\right] .
$$

Since $i_{1} \in X_{n}(A) \backslash\{i\}$, then, by the property of $A$, there exists $i_{2} \in X_{n}(A) \backslash\left\{i_{1}\right\}$ such that

$$
\alpha_{i_{2}} \in A \text { and }\left[i_{1} \rightarrow i_{1}+1\right] \mathcal{L} \alpha_{i_{2}} \mathcal{R}\left[i_{2} \rightarrow i_{2}+1\right] .
$$

If $i_{2}=i$, then, by Lemma 2.2,

$$
\alpha_{i_{2}}^{*}=\alpha_{i_{2}} \alpha_{i_{1}} \in H_{\left(i_{2}, i_{2}+1\right)}^{\left[i_{1}\right]} H_{\left(i_{1}, i_{1}+1\right)}^{[i]}=H_{\left(i_{2}, i_{2}+1\right)}^{[i]}=H_{(i, i+1)}^{[i]} .
$$

Since $[i \rightarrow i+1]$ is an idempotent of the group $H_{(i, i+1)}^{[i]}$, it follows that $[i \rightarrow$ $i+1]=\left(\alpha_{i_{2}}^{*}\right)^{\omega}$ for some $\omega \in \mathbb{N}$, and so $[i \rightarrow i+1] \in\langle A\rangle$. Notice that $i_{2} \in X_{n}(A) \backslash\left\{i_{1}\right\}$. If $i_{2} \neq i$, then, by the property of $A$, there exists $i_{3} \in$ $X_{n}(A) \backslash\left\{i_{1}, i_{2}\right\}$ such that

$$
\alpha_{i_{3}} \in A \text { and }\left[i_{2} \rightarrow i_{2}+1\right] \mathcal{L} \alpha_{i_{3}} \mathcal{R}\left[i_{3} \rightarrow i_{3}+1\right] .
$$

Notice that $i \in X_{n}(A)$. Continuing this demonstration, by the property of $A$, there exist distinct $i_{1}, i_{2}, \ldots, i_{m-1}, i_{m} \in X_{n}(A)\left(m \leq\left|X_{n}(A)\right|\right)$ such that $i_{m}=i$ and

$$
\begin{gathered}
\alpha_{i_{1}} \in A \text { and }[i \rightarrow i+1] \mathcal{L} \alpha_{i_{1}} \mathcal{R}\left[i_{1} \rightarrow i_{1}+1\right], \\
\alpha_{i_{k}} \in A \text { and }\left[i_{k-1} \rightarrow i_{k-1}+1\right] \mathcal{L} \alpha_{i_{k}} \mathcal{R}\left[i_{k} \rightarrow i_{k}+1\right],
\end{gathered}
$$

for $k \in\{2, \ldots, m\}$. Then, by Lemma 2.2,

$$
\begin{aligned}
\alpha_{i_{m}}^{*} & =\alpha_{i_{m}} \alpha_{i_{m-1}} \cdots \alpha_{i_{1}} \\
& \in H_{\left(i_{m}, i_{m}+1\right)}^{\left[i_{m-1}\right]} H_{\left(i_{m-1}, i_{m-1}+1\right)}^{\left[i_{m-2}\right]} \cdots H_{\left(i_{3}, i_{3}+1\right)}^{\left[i_{2}\right]} H_{\left(i_{2}, i_{2}+1\right)}^{\left[i_{1}\right]} H_{\left(i_{1}, i_{1}+1\right)}^{[i]}
\end{aligned}
$$




$$
=H_{\left(i_{m}, i_{m}+1\right)}^{[i]}=H_{(i, i+1)}^{[i]} .
$$

Since $[i \rightarrow i+1]$ is an idempotent of the group $H_{(i, i+1)}^{[i]}$, it follows that $[i \rightarrow$ $i+1]=\left(\alpha_{i_{m}}^{*}\right)^{\omega}$ for some $\omega \in \mathbb{N}$, and so $[i \rightarrow i+1] \in\langle A\rangle$.

Since $\mathcal{S O P}_{n}$ has rank $n$ (see [8, Theorem 2.2]), a generating set of $\mathcal{S O P}_{n}$ with $n$ elements is a minimal generating set. Moreover, if $\alpha$ is an element of $\mathcal{S O P}{ }_{n}$ of rank $n-1$ and $\beta$ and $\gamma$ are two elements of $\mathcal{S O P}{ }_{n}$ such that $\alpha=\beta \gamma$, then $\operatorname{ker}(\alpha)=\operatorname{ker}(\beta)$ and $\operatorname{im}(\alpha)=\operatorname{im}(\gamma)$. Then any generating set of $\mathcal{S O P}{ }_{n}$ with $n$ elements must be the subset having exactly one element from each $\mathcal{R}$-class and from each $\mathcal{L}$-class of $\mathcal{S O P}_{n}$ contained in $[n, n-1]$. These observations, together with the Lemma 2.3, prove the following result:

Theorem 2.4. Let $n \geq 3$, and let $M$ be a nonempty subset of $\mathcal{S O P}_{n}$ with $n$ elements. Then $M$ is a minimal generating set of $\mathcal{S O P}_{n}$ if and only if $M$ be the subset having exactly one element from each $\mathcal{R}$-class and from each $\mathcal{L}$-class of $\mathcal{S O P}_{n}$ contained in $[n, n-1]$.

Notice also that each $\mathcal{H}$-class of $\mathcal{S O P}_{n}$ contained in $[n, n-1]$ has $n-1$ elements (see [2, Corollary 3.6]). Thus we have the following corollary from Theorem 2.4:

Corollary 2.5. Let $n \geq 3$, and let $M$ be a minimal generating set of $\mathcal{S O P}_{n}$. Then the number of distinct sets $M$ is $(n-1)^{n} n$ !.

Now, consider the permutation (n-cycle) $g=\left(\begin{array}{ccccc}1 & 2 & \cdots & n-1 & n \\ 2 & 3 & \cdots & n & 1\end{array}\right)$ of $X_{n}$. For $1 \leq s \leq n-1$ and $1 \leq i \leq n$, let

$$
\alpha_{(1,2)}^{[s+1]}=\left(\begin{array}{ccccccc}
\{1,2\} & 3 & \cdots & s+1 & s+2 & \cdots & n \\
1 & 2 & \cdots & s & s+2 & \cdots & n
\end{array}\right) .
$$

and

$$
\alpha_{(i+1, i+2)}^{[s+1]}=g^{-i} \alpha_{(1,2)}^{[s+1]} g^{i} .
$$

Then clearly $\alpha_{(1,2)}^{[s+1]} \in H_{(1,2)}^{[s+1]}$. Notice also that $\alpha_{(n+1, n+2)}^{[s+1]}=\alpha_{(1,2)}^{[s+1]}$.

Lemma 2.6. Let $1 \leq s \leq n-1$ and $1 \leq i \leq n$. Then $\alpha_{(i+1, i+2)}^{[s+1]} \in H_{(i+1, i+2)}^{[i+s+1]}$ and $\alpha_{(i+1, i+2)}^{[s+1]}$ is s-potent.

Proof. Since $g$ is a permutation $\left(n\right.$-cycle) of $X_{n}$ and $[1 \rightarrow 2] \mathcal{R} \alpha_{(1,2)}^{[s+1]} \mathcal{L}[s+1 \rightarrow$ $s+2]$, we have

$$
g^{-i}[1 \rightarrow 2] \mathcal{R} g^{-i} \alpha_{(1,2)}^{[s+1]} \text { and } \alpha_{(1,2)}^{[s+1]} g^{i} \mathcal{L}[s+1 \rightarrow s+2] g^{i} .
$$

Notice that

$g^{-i}[1 \rightarrow 2] g^{i}=[i+1 \rightarrow i+2], g^{-i}[s+1 \rightarrow s+2] g^{i}=[i+s+1 \rightarrow i+s+2]$ 
and $\mathcal{R}(\mathcal{L})$ is a right (left) congruence. Then

$$
\alpha_{(i+1, i+2)}^{[s+1]}=g^{-i} \alpha_{(1,2)}^{[s+1]} g^{i} \mathcal{R} g^{-i}[1 \rightarrow 2] g^{i}=[i+1 \rightarrow i+2]
$$

and

$$
\alpha_{(i+1, i+2)}^{[s+1]}=g^{-i} \alpha_{(1,2)}^{[s+1]} g^{i} \mathcal{L} g^{-i}[s+1 \rightarrow s+2] g^{i}=[i+s+1 \rightarrow i+s+2] .
$$

Thence $\alpha_{(i+1, i+2)}^{[s+1]} \in H_{(i+1, i+2)}^{[i+s+1]}$.

Notice that $\alpha_{(1,2)}^{[2]}$ is an idempotent and $g^{i} g^{-i}=1_{X_{n}}$. Then

$$
\left(\alpha_{(i+1, i+2)}^{[2]}\right)^{2}=g^{-i}\left(\alpha_{(1,2)}^{[2]}\right)^{2} g^{i}=g^{-i} \alpha_{(1,2)}^{[2]} g^{i}=\alpha_{(i+1, i+2)}^{[2]}
$$

and so $\alpha_{(i+1, i+2)}^{[2]}$ is 1 -potent. Notice also that, for $2 \leq s \leq n-1$,

$$
\begin{aligned}
& \left(\alpha_{(i+1, i+2)}^{[s+1]}\right)^{k} \\
= & g^{-i}\left(\alpha_{(1,2)}^{[s+1]}\right)^{k} g^{i} \\
= & g^{-i}\left(\begin{array}{ccccccc}
\{1,2, \cdots, k+1\} & k+2 & \cdots & s+1 & s+2 & \cdots & n \\
1 & 2 & \cdots & s+1-k & s+2 & \cdots & n
\end{array}\right) g^{i}, \\
& 1 \leq k \leq s-1
\end{aligned}
$$

and

$$
\left(\alpha_{(i+1, i+2)}^{[s+1]}\right)^{s}=g^{-i}\left(\begin{array}{cccc}
\{1,2, \cdots, s+1\} & s+2 & \cdots & n \\
1 & s+2 & \cdots & n
\end{array}\right) g^{i} .
$$

Then $\left(\alpha_{(i+1, i+2)}^{[s+1]}\right)^{s+1}=\left(\alpha_{(i+1, i+2)}^{[s+1]}\right)^{s}$ and

$$
\left(\alpha_{(i+1, i+2)}^{[s+1]}\right)^{1},\left(\alpha_{(i+1, i+2)}^{[s+1]}\right)^{2}, \ldots,\left(\alpha_{(i+1, i+2)}^{[s+1]}\right)^{s}
$$

are distinct. Hence $\alpha_{(i+1, i+2)}^{[s+1]}$ is $s$-potent.

Now, for $1 \leq s \leq n-1$, let

$$
G(s)=\left\{\alpha_{(i+1, i+2)}^{[s+1]}: 1 \leq i \leq n\right\} .
$$

Then, by Lemma 2.6 , the set $G(s)$ contains exactly one element from each $\mathcal{R}$ class and from each $\mathcal{L}$-class of $\mathcal{S O P}{ }_{n}$ contained in $[n, n-1]$. Thus, by Theorem $2.4, \mathcal{S O P}_{n}=\langle G(s)\rangle$. Notice that $|G(s)|=n$ and the $s$-potent rank of $\mathcal{S O P}_{n}$ is at least as large as the rank of $\mathcal{S O P}{ }_{n}$. Recall that the rank and the idempotent rank of $\mathcal{S O P}_{n}$ are both equal to $n$ (see [8, Theorem 2.2]). These observations, together with Lemma 2.6, prove the following result:

Theorem 2.7. Let $1 \leq m \leq n-1$. Then $\operatorname{rank}_{m} \mathcal{S O P}_{n}=n$.

Now, recall that Zhao [8, Theorem 2.3] proved:

Lemma 2.8. Let $n \geq 3$. Let $G \subseteq E\left(\mathcal{S P O P}{ }_{n}\right)$. Then

$\langle G\rangle=\mathcal{S P O P} \mathcal{P}_{n}$ if and only if $E_{n-1}^{+} \cup F_{n-1} \subseteq G$ or $E_{n-1}^{-} \cup F_{n-1} \subseteq G$.

Using Lemma 2.8, it is easy to prove the following result: 
Lemma 2.9. Let $n \geq 3$. Let $A$ be a subset of $[n-1, n-1]$. If $A$ contains one element from each $\mathcal{R}$-class of $\mathcal{S P O P}{ }_{n}$ contained in $[n-1, n-1]$, then $\mathcal{S P O P}{ }_{n}=\left\langle E_{n-1}^{+} \cup A\right\rangle$.

Proof. By Lemma 2.8, we have $\mathcal{S P O P}{ }_{n}=\left\langle E_{n-1}^{+} \cup F_{n-1}\right\rangle$. We shall show that $F_{n-1} \subseteq\left\langle E_{n-1}^{+} \cup A\right\rangle$ and so $\mathcal{S P O P}{ }_{n}=\left\langle E_{n-1}^{+} \cup A\right\rangle$. Let $\delta_{i} \in F_{n-1}$, then there exists $\alpha \in A$ such that $\alpha \mathcal{R} \delta_{i}$. Then $\alpha \in R_{i}$. Suppose that $\alpha \in H_{(i)}^{[k]}$ for some $k \in\{1,2, \ldots, n\}$. Let $\beta \in H_{(k, k+1)}^{[i]}$. Then, by Lemmas 2.1 and $2.2, \beta \in \mathcal{S O P}_{n}=\left\langle E_{n-1}^{+}\right\rangle$and $\alpha \beta \in H_{(i)}^{[k]} H_{(k, k+1)}^{[i]}=H_{(i)}^{[i]}$. Since $\delta_{i}$ is an idempotent of the group $H_{(i)}^{[i]}$, it follows that $\delta_{i}=(\alpha \beta)^{\omega}$ for some $\omega \in \mathbb{N}$, whence $\delta_{i} \in\left\langle E_{n-1}^{+} \cup A\right\rangle$.

For $1 \leq s \leq n-1$ and $1 \leq i \leq n$, let

$$
\beta_{[1, s+1]}=\left(\begin{array}{ccccccc}
2 & 3 & \cdots & s+1 & s+2 & \cdots & n \\
1 & 2 & \cdots & s & s+2 & \cdots & n
\end{array}\right)
$$

and

$$
\beta_{[i+1, s+1]}=g^{-i} \beta_{[1, s+1]} g^{i} .
$$

Then clearly $\beta_{[1, s+1]} \in H_{(1)}^{[s+1]}$. Notice also that $\beta_{[n+1, s+1]}=\beta_{[1, s+1]}$.

Lemma 2.10. Let $1 \leq s \leq n-1$ and $1 \leq i \leq n$. Then $\beta_{[i+1, s+1]} \in H_{(i+1)}^{[i+s+1]}$ and $\beta_{[i+1, s+1]}$ is $(s+1)$-potent.

Proof. Since $g$ is a permutation ( $n$-cycle) of $X_{n}$ and $\delta_{1} \mathcal{R} \beta_{[1, s+1]} \mathcal{L} \delta_{s+1}$, we have

$$
g^{-i} \delta_{1} \mathcal{R} g^{-i} \beta_{[1, s+1]} \text { and } \beta_{[1, s+1]} g^{i} \mathcal{L} \delta_{s+1} g^{i} .
$$

Notice that

$$
g^{-i} \delta_{1} g^{i}=\delta_{i+1}, g^{-i} \delta_{s+1} g^{i}=\delta_{i+s+1}
$$

and $\mathcal{R}(\mathcal{L})$ is a right (left) congruence. We mean $\delta_{n+j}=\delta_{j}$ for $j=1,2, \ldots, n$ by Remark 1 . Then

$$
\begin{aligned}
& \beta_{[i+1, s+1]}=g^{-i} \beta_{[1, s+1]} g^{i} \mathcal{R} g^{-i} \delta_{1} g^{i}=\delta_{i+1}, \\
& \beta_{[i+1, s+1]}=g^{-i} \beta_{[1, s+1]} g^{i} \mathcal{L} g^{-i} \delta_{s+1} g^{i}=\delta_{i+s+1} .
\end{aligned}
$$

Thence $\beta_{[i+1, s+1]} \in H_{(i+1)}^{[i+s+1]}$.

Notice that $\beta_{[1,2]}=\left(\begin{array}{cccc}2 & 3 & \cdots & n \\ 1 & 3 & \cdots & n\end{array}\right)$ and $g^{i} g^{-i}=1_{X_{n}}$. Then

$$
\left(\beta_{[i+1,2]}\right)^{2}=g^{-i}\left(\beta_{[1,2]}\right)^{2} g^{i}=g^{-i}\left(\begin{array}{ccc}
3 & \cdots & n \\
3 & \cdots & n
\end{array}\right) g^{i} .
$$

Then $\left(\beta_{[i+1,2]}\right)^{3}=\left(\beta_{[i+1,2]}\right)^{2}$ and $\left(\beta_{[i+1,2]}\right)^{1},\left(\beta_{[i+1,2]}\right)^{2}$ are distinct. Hence $\beta_{[i+1,2]}$ is 2-potent. Notice also that, for $2 \leq s \leq n-1$,

$$
\left(\beta_{[i+1, s+1]}\right)^{k}
$$




$$
\begin{aligned}
& =g^{-i}\left[\beta_{[1, s+1]}\right]^{k} g^{i} \\
& =g^{-i}\left(\begin{array}{ccccccc}
k+1 & k+2 & \cdots & s+1 & s+2 & \cdots & n \\
1 & 2 & \cdots & s+1-k & s+2 & \cdots & n
\end{array}\right) g^{i}, 1 \leq k \leq s
\end{aligned}
$$

and

$$
\left(\beta_{[i+1, s+1]}\right)^{s+1}=g^{-i}\left(\begin{array}{ccc}
s+2 & \cdots & n \\
s+2 & \cdots & n
\end{array}\right) g^{i} .
$$

Then $\left(\beta_{[i+1, s+1]}\right)^{s+2}=\left(\beta_{[i+1, s+1]}\right)^{s+1}$ and

$$
\left(\beta_{[i+1, s+1]}\right)^{1},\left(\beta_{[i+1, s+1]}\right)^{2}, \ldots,\left(\beta_{[i+1, s+1]}\right)^{s+1}
$$

are distinct. Hence $\beta_{[i+1, s+1]}$ is $(s+1)$-potent.

For $1 \leq s \leq n-1$, let

$$
F(s)=\left\{\beta_{[i+1, s+1]}: 1 \leq i \leq n\right\} .
$$

Then, by Lemma 2.10 , the set $F(s)$ contains exactly one element from each $\mathcal{R}$-class and from each $\mathcal{L}$-class of $\mathcal{S P O P}{ }_{n}$ contained in $[n-1, n-1]$. Thus, by Theorem 2.4 and Lemma $2.9, \mathcal{S P O P}_{n}=\langle G(s+1) \cup F(s)\rangle$ for $1 \leq s \leq n-2$. Notice that $|G(s+1) \cup F(s)|=2 n$ and the $(s+1)$-potent rank of $\mathcal{S P O P} \mathcal{P}_{n}$ is at least as large as the rank of $\mathcal{S P O P}{ }_{n}$. Recall that the rank and the idempotent rank of $\mathcal{S P O P}{ }_{n}$ are both equal to $2 n$ (see [8, Theorem 2.9]). These observations, together with Lemmas 2.6 and 2.10, prove the following result:

Theorem 2.11. Let $1 \leq m \leq n-1$. Then $\operatorname{rank}_{m} \mathcal{S P O P}{ }_{n}=2 n$.

\section{The 2-potent rank of $\mathcal{S S P \mathcal { P }}{ }_{n}$}

The subset $\mathcal{S S P O P}{ }_{n}=\mathcal{S P O P}{ }_{n} \backslash \mathcal{S O P}{ }_{n}$ of strictly partial orientation preserving mappings on $X_{n}$ is a subsemigroup of $\mathcal{S P O P}{ }_{n}$. Since a non-idempotent element in $[n-1, n-1]$ can not be expressed as a product of idempotents of $[n-1, n-1]$, the semigroup $\mathcal{S S P O P P}{ }_{n}$ is not idempotent-generated. Then $\mathcal{S} \mathcal{S P O P}{ }_{n}$ has no 1-potent rank.

Let

$$
\begin{aligned}
\beta & =\left(\begin{array}{llll}
1 & 2 & \cdots & n-1 \\
2 & 2 & \cdots & n-1
\end{array}\right), \\
\alpha_{1} & =\left(\begin{array}{lllll}
2 & 3 & \cdots & n-1 & n \\
2 & 3 & \cdots & n-1 & 1
\end{array}\right) \in H_{(1)}^{[n]}
\end{aligned}
$$

and

$$
\alpha_{i}=\left(\begin{array}{cccccccc}
1 & \cdots & i-2 & i-1 & i+1 & i+2 & \cdots & n \\
1 & \cdots & i-2 & i & i+1 & i+2 & \cdots & n
\end{array}\right) \in H_{(i)}^{[i-1]}
$$

for $2 \leq i \leq n$. Recall that Zhao [8, Lemma 3.3 and Lemma 3.8] proved:

Lemma 3.1. Let $B=\left\{\alpha_{i}: 1 \leq i \leq n\right\}$ and $G=B \cup\{\beta\}$. Then $[n-1, n-1] \subseteq$ $\langle B\rangle$ and $\mathcal{S} \mathcal{S P O P}{ }_{n}=\langle G\rangle$. 
Now, let

$$
\widetilde{\beta}=\left(\begin{array}{cccccc}
1 & 2 & 3 & \cdots & n-2 & n-1 \\
n & 2 & 3 & \cdots & n-2 & n
\end{array}\right) .
$$

Then $\widetilde{\beta}$ is 2-potent.

Lemma 3.2. Let $B=\left\{\alpha_{i}: 1 \leq i \leq n\right\}$ and $\widetilde{G}=B \cup\{\widetilde{\beta}\}$. Then $\mathcal{S S P O P}_{n}=$ $\langle\widetilde{G}\rangle$.

Proof. Notice that $\alpha_{i} \in[n-1, n-1]$, for $1 \leq i \leq n$. Then, by Lemma 3.1, $\mathcal{S S P O P}{ }_{n}=\langle[n-1, n-1] \cup\{\beta\}\rangle$. Now, as

$$
\begin{aligned}
\beta & =\left(\begin{array}{cccccc}
1 & 2 & 3 & \cdots & n-2 & n-1 \\
n-1 & 1 & 2 & \cdots & n-3 & n-2
\end{array}\right) \widetilde{\beta}\left(\begin{array}{cccccc}
2 & 3 & \cdots & n-2 & n-1 & n \\
3 & 4 & \cdots & n-1 & n & 2
\end{array}\right) \\
& \in\langle[n-1, n-1] \cup\{\widetilde{\beta}\}\rangle,
\end{aligned}
$$

it follows that $\mathcal{S S P O P}{ }_{n}=\langle[n-1, n-1] \cup\{\widetilde{\beta}\}\rangle$. Then, again by Lemma 3.1, $\mathcal{S} \mathcal{S P O P}{ }_{n}=\langle\widetilde{G}\rangle$.

Notice that $|\widetilde{G}|=n+1$, each element of the set $\widetilde{G}$ is 2 -potent and the 2 potent rank of $\mathcal{S} \mathcal{S P O P}{ }_{n}$ is at least as large as the rank of $\mathcal{S} \mathcal{S P O P}{ }_{n}$. Recall that the rank of $\mathcal{S S P O P}{ }_{n}$ is equal to $n+1$ (see [8, Theorem 3.1]). These observations, together with Lemma 3.2, prove the following result:

Theorem 3.3. Let $n \geq 3$. Then $\operatorname{rank}_{2} \mathcal{S} \mathcal{S P O P} \mathcal{P}_{n}=n+1$.

Acknowledgments. The authors would like to thank the anonymous referee for his/her helpful comments and suggestions that helped to improve this paper.

\section{References}

[1] G. Ayık, H. Ayık, Y. Ünlü, and J. M. Howie, The structure of elements in finite full transformation semigroups, Bull. Aust. Math. Soc. 71 (2005), no. 1, 69-74.

[2] P. M. Catarino and P. M. Higgins, The monoid of orientation-preserving mappings on a chain, Semigroup Forum 58 (1999), no. 2, 190-206.

[3] V. H. Fernandes, G. M. S. Gomes, and M. M. Jesus, Congruences on monoids of transformations preserving the orientation on a finite chain, J. Algebra 321 (2009), no. 3, 743-757.

[4] G. M. S. Gomes and J. M. Howie, On the ranks of certain semigroups of order-preserving transformations, Semigroup Forum 45 (1992), no. 3, 272-282.

[5] J. M. Howie, Fundamentals of Semigroup Theory, Oxford, Oxford University Press, 1995.

[6] O. Sönmez, Combinatorics of elements in $T_{n}$ and $P T_{n}$, Int. J. Algebra 4 (2010), no. 1-4, $45-51$.

[7] O. Sönmez and Y. Ünlü, m-potent elements in order-preserving transformation semigroups and ordered trees, Comm. Algebra 42 (2014), no. 1, 332-342.

[8] P. Zhao, On the ranks of certain semigroups of orientation preserving transformations, Comm. Algebra 39 (2011), no. 11, 4195-4205.

[9] P. Zhao, X. Bo, and Y. Mei, Locally maximal idempotent-generated subsemigroups of singular orientation-preserving transformation semigroups, Semigroup Forum 77 (2008), no. $2,187-195$. 
Ping ZhaO

School of Mathematics and Computer Science

Guizhou Normal University

Guiyang, Guizhou Province 550001, P. R. China

AND

Mathematics Teaching \& Research Section

Guiyang Medical College

Guiyang, Guizhou Province 550004, P. R. China

E-mail address: zhaoping731108@hotmail.com

TAIJIE You

School of Mathematics and Computer Science

Guizhou Normal University

Guiyang, Guizhou Province 550001, P. R. China

E-mail address: taijieyou@163.com

HuABI Hu

Department of Medicine Bioengineering

Guiyang Medical College

Guiyang, Guizhou Province 550004, P. R. China

E-mail address: huhuabi@hotmail.com 